\begin{tabular}{|c|l|}
\hline Title & The bactericidal effect of carbon nanotube/agar composites irradiated with near-infrared light on Streptococcus mutans \\
\hline Author(s) & A kasaka, T sukasa; Matsuoka, Makoto; Hashimoto, Takeshi; A be, Shigeaki; Uo, Motohiro; W atari, Fumio \\
\hline Citation & $\begin{array}{l}\text { Materials Science and Engineering: B, 173(1-3), 187-190 } \\
\text { https://doi.org/10.1016/.mseb.2010.01.001 }\end{array}$ \\
\hline Issue Date & 2010 -10 \\
\hline Doc URL & http://hdl.handle.net/2115/48564 \\
\hline Type & article(author version) \\
\hline File Information & A kasaka20120314_3.pdf \\
\hline
\end{tabular}

Instructions for use 


\section{The bactericidal effect of carbon nanotube/agar composites irradiated with near-infrared light on Streptococcus mutans}

Tsukasa Akasaka ${ }^{\mathrm{a}, *}$, Makoto Matsuoka ${ }^{\mathrm{a}}$, Takeshi Hashimoto ${ }^{\mathrm{b}}$, Shigeaki Abe ${ }^{\mathrm{a}}$, Motohiro Uo ${ }^{\text {a }}$, Fumio Watari ${ }^{\text {a }}$

${ }^{a}$ Graduate School of Dental Medicine, Hokkaido University, Kita13 Nishi7, Kita-ku, Sapporo 060-8586, Japan

${ }^{\text {b }}$ Meijo Nano Carbon Co. Ltd., Otsubashi bldg. 4F, 3-4-10 Marunouchi, Naka-ku, Nagoya 460-0002, Japan

* Corresponding author: Department of Biomedical, Dental Materials and Engineering, Graduate School of Dental Medicine, Hokkaido University, Kita13 Nishi7, Kita-ku, Sapporo 060-8586, Japan.

Tel.: +81 11706 4251; fax: +81 117064251 .

E-mail address: akasaka@den.hokudai.ac.jp.

\section{Abstract}

Dental caries are mainly associated with oral pathogens, and Streptococcus 
mutans is a primary cariogenic organism. Many methods have been established to eliminate $S$. mutans from the oral cavity. This study aimed to evaluate the effect of carbon nanotube (CNT)/agar composites irradiated with near-infrared (NIR) light on $S$. mutans, as a potential photothermal antimicrobial nanotherapy. A colony-forming unit assay clearly showed that CNT/agar composites attain bactericidal activity after NIR light irradiation; this bactericidal activity is higher than that of graphite (GP)/agar and activated carbon (AC)/agar composites. Furthermore, it was observed that longer irradiation times immobilized S. mutans in the CNT/agar composite.

Keywords: Carbon nanotubes; Streptococcus mutans; bactericidal activity; near-infrared light 


\section{Introduction}

The occurrence of dental caries is mainly associated with oral pathogens, and Streptococcus mutans is a primary cariogenic organism. Therefore, many methods have been established to eliminate $S$. mutans from the oral cavity [1,2]. One such approach is photodynamic therapy [3-5]. A series of studies have demonstrated the photodynamic inactivation of bacteria by the exogenous application of a photosensitizer such as methylene blue or toluidine blue [4].

Carbon nanotubes (CNTs) have attracted considerable attention because of their unique physical properties and potential for a variety of biological applications [6,7]. In addition, CNTs can adsorb bacteria [8-11]. Single-walled carbon nanotubes (SWNTs) exert strong antimicrobial activity against Escherichia coli [12,13]. Kim et al reported novel applications of CNTs in photothermal antimicrobial nanotherapy [14]. Their study showed the excellent capability of SWNTs and multi-walled carbon nanotubes (MWNTs) to serve as near-infrared (NIR)-responsive clustering photothermal contrast agents. By NIR light irradiation, CNTs are promoted to the electronic excited state, followed by rapid relaxation to the ground state with an effective electron-phonon conversion of the absorbed photon energy into thermal energy.

We reported that MWNTs had the highest efficiency of precipitating $S$. mutans 
among the carbon materials, such as CNTs and activated carbon (AC) [11]. It has been shown that carbon materials with different diameters and sizes have significantly different effects on the precipitation efficiency and the manner of capture of $S$. mutans. However, the bactericidal effect of CNTs irradiated with NIR light and, particularly, the dose-dependent efficacy of CNTs in comparison with other carbon materials have not yet been sufficiently investigated. The aim of this study was to evaluate the bactericidal effect of CNT/agar composites irradiated with NIR light on S. mutans, as a potential photothermal antimicrobial nanotherapy. The dose-dependent efficacy of MWNTs in the composites was investigated by a colony-forming unit (CFU) assay. Subsequently, the effect of different types of carbon materials such as SWNTs, MWNTs, graphite (GP), and AC was also investigated. The CFU assay with agar composites containing carbon materials is useful for studying the photothermal effect, except the difference in the efficacy of bacterial adhesion of carbon materials. We also attempted to observe the morphology of the bacteria in the CNT/agar composites irradiated with NIR light.

\section{Experimental}

The purified SWNTs $(0.8-2.5 \mathrm{~nm}$ in diameter) used in this study were purchased from Meijo Nano Carbon Co. Ltd. (Nagoya, Japan) and synthesized by an arc discharge 
method [15]; the MWNTs (20-40 nm in diameter) were purchased from NanoLab Inc. (Brighton, MA). The MWNTs were purified using a previously reported method [16]. The SWNTs had a purity of $>95 \mathrm{wt} \%$ and contained $<5 \mathrm{wt} \%$ amorphous carbon as the dominant impurity, whereas the MWNTs had a purity of $>98 \mathrm{wt} \%$ and contained $<2$ wt\% amorphous carbon. Commercial AC powder with an average particle size of $<20$ $\mu \mathrm{m}$ (Kanto Chemical Co. Inc., Tokyo, Japan) and synthetic GP with an average particle size of $<20 \mu \mathrm{m}$ (Sigma-Aldrich Co. Ltd., St. Louis, MO) were used as the control carbon materials in this study. To prepare the CNT/agar composites, a suspension of CNTs (0.1 mg/ml and $1.0 \mathrm{mg} / \mathrm{ml})$ and brain heart infusion (BHI) agar (Pearlcore; Eiken Chemical Co. Ltd., Tokyo, Japan) in $\mathrm{H}_{2} \mathrm{O}$ was dispersed by ultrasonication, followed by microwave heating. A $10-\mathrm{ml}$ aliquot of the dispersed solution was poured into a 100-mm culture dish and was left undisturbed until the CNT/agar composites solidified.

Macroscopic fields of the CNT/agar composites molded to a square of $1 \times 1 \times 1$ $\mathrm{cm}^{3}$ were photographed. The luminous transparency of 0.2 -mm-thick blocks of the composites was measured using a luminous transmittance meter (STS-4; Fujikoden Corp., Kanagawa, Japan).

S. mutans JC2 strains were used in this study. S. mutans was grown in BHI broth at $37^{\circ} \mathrm{C}$ for several days under aerobic conditions. The bacteria were harvested by 
centrifugation at $2500 \times g$ (Kubota Centrifuge 2700, Tokyo, Japan) and suspended in BHI broth at a concentration of $6.8 \times 10^{3} \mathrm{CFU} / \mathrm{ml}$. The CNT/agar composites were then inoculated with $5 \mu \mathrm{l}$ of the $S$. mutans culture solution, followed by irradiation with NIR light. Irradiation was performed using NIR light (LA-100IR; Hayashi Watch Works Co. Ltd., Tokyo, Japan) with a light power of $1.3 \times 10^{4} \mathrm{~W} / \mathrm{m}^{2}$ or $5.6 \times 10^{3} \mathrm{~W} / \mathrm{m}^{2}$ at a wavelength of $800-1300 \mathrm{~nm}$. The agar plates were incubated for $48 \mathrm{~h}$ at $37^{\circ} \mathrm{C}$ under anaerobic condition, and the colonies were then counted. Student's $t$ test was used to assess the statistical significance of the results. All statistical analyses were performed using Microsoft Excel at a confidence level of 95\%.

For scanning electron microscope (SEM) observation, the CNT/agar composites were fixed with a $2.5 \%$ solution of glutaraldehyde and postfixed in $1 \%$ osmium tetroxide. The composites were dehydrated with a graded alcohol series $(50 \%, 70 \%$, $80 \%, 90 \%$, 95\%, and $100 \%$ ), subjected to critical point drying at $37^{\circ} \mathrm{C}$, and coated with Pt/Pd. The SEM images were obtained using a Hitachi S-4000 SEM.

\section{Results and discussion}

The CNT/agar composites formed by the aqueous dispersion of CNTs and agar powder were compared to agar gel without CNTs (Fig. 1a). The CNT/agar composites 
were soft and black. The luminous transmittance of 0.2 -mm-thick blocks of the CNT/agar composites was measured using a luminous transmittance meter (Fig. 1b). The luminous transmittance of the CNT/agar composites decreased with increasing loading of CNTs $(0.1-\mathrm{mg} / \mathrm{ml}$ to $1.0-\mathrm{mg} / \mathrm{ml})$ and ranged from of $81 \%$ to $31 \%$. The GP/agar and AC/agar composites had a higher transmittance ranging from $84 \%$ to $60 \%$ because the particle sizes of GP and AC were larger than those of the CNTs. As a result, the GP and AC particles covered a small surface area and were not completely dispersed in the agar gel.

The bactericidal activities of the MWNT/agar composites irradiated with NIR light were assessed by a CFU assay. The results of the assay depend on the amount of MWNTs contained in the agar (Fig. 2). Colony formation on normal agar did not depend on irradiation time; however, in colony formation on the MWNT/agar composites was observed to reduce with longer irradiation times. Further, the decrease in the CFUs was greater in composites with $1.0-\mathrm{mg} / \mathrm{ml}$ loading than those with 0.1-mg/ml loading. The results clearly show that MWNT/agar composites irradiated with NIR light exert bactericidal activity. Kim et al reported that clustered CNTs have higher bactericidal activity than individual CNTs [14]. Therefore, the MWNT/agar composite with $1.0-\mathrm{mg} / \mathrm{ml}$ loading may have higher bactericidal activity owing to the 
strong absorption of NIR light.

Figure 3a shows that the number of CFUs on the carbon/agar composites irradiated with NIR light depends on the type of carbon material used. The percentage of CFUs on the various carbon/agar composites irradiated with NIR light was compared to that of CFUs on normal agar (Fig. 3a). The percentage of live bacteria decreased on all carbon/agar composites, with reductions of ca. $100 \%$ after 60 -s irradiation. The bactericidal activity of the CNT/agar composites was higher than that of GP/agar and AC/agar composites at 30-s irradiation $(p<0.05)$. MWNT/agar and SWNT/agar composites resulted in the highest decreases of $100 \%$ each after 30 -s irradiation, whereas GP/agar and AC/agar composites resulted in decreases of $66 \%$ and $60 \%$ after 30-s irradiation, respectively (Fig. 3b). The bactericidal activities of GP/agar and AC/agar were low owing to their high luminous transmittance (Fig. 1b), which resulted in weak absorption of NIR light. This result shows that the bactericidal activities of MWNT/agar and SWNT/agar composites were higher than those of GP/agar and AC/agar composites irradiated with NIR light. The similar bactericidal efficacy of the MWNT/agar and SWNT/agar composites against $S$. mutans observed in our study was in agreement with the efficacy of NIR-irradiated MWNT and SWNT clusters against E. coli [14]. The bactericidal activity of the CNTs irradiated with NIR light is possibly 
dependent on the transmittance of CNT composites rather than their structures, with certain variables such as the wavelength of the NIR light used.

Subsequently, the bacteria on the MWNT/agar composite irradiated with NIR light for different durations were observed using an SEM. Figure 4 shows the SEM image of S. mutans on MWNT/agar composites or normal agar. Several bacteria (radius of the minor axis, $500 \mathrm{~nm}$ ) were observed on the meshwork of agar. Fibrous polymeric substances with a width of about 20-40 nm were observed in the agar. In Figure 4a-c, the MWNTs and fibrous agar could not be clearly distinguished using the SEM. Changes in the morphology of $S$. mutans induced by NIR light were not observed. The mechanism underlying the bactericidal effect on $S$. mutans possibly involves the denaturation of bacterial proteins and not the destruction of the cell wall because of the heat generated by NIR light irradiation.

Furthermore, it was observed that longer irradiation time, i.e., 120 s, caused the S. mutans to become imbedded in the MWNT/agar composite (Fig. 4c). In normal agar used as control, we did not observe changes in the morphology of $S$. mutans on the agar induced by NIR light (Fig. 4d-f). Prolonged NIR light irradiation caused greater heating of the agar containing the CNTs, following which the agar melted, thus, immobilizing $S$. mutans bacteria. Prolonged irradiation of the CNT/agar composite may be 
advantageous not only for bacterial killing but also for immobilizing S. mutans in the agar. However, further investigations are required to determine the effects of the size of carbon materials and the wavelength of NIR light on the bactericidal activity of the CNT/agar composites against S. mutans.

\section{Conclusion}

The CNT/agar composites have potential applications as photothermal antibiotic materials that immobilize bacteria in the agar depending on the irradiation conditions. Our results clearly show that CNT/agar composites exhibit bactericidal activity when irradiated with NIR light. The bactericidal effect exerted against $S$. mutans possibly involves the denaturation of bacterial proteins and not the collapse of the cell wall because of the heat generated by strong absorption of NIR light by the CNTs. The NIR-irradiated CNT/agar composites possess greater bactericidal activity than the GP/agar and AC/agar composites. Furthermore, it was observed that a longer irradiation time, i.e., $120 \mathrm{~s}$, immobilized S. mutans in the MWNT/agar composite. Therefore, CNT/agar composites may be used for the photodynamic inactivation of oral pathogens and the elimination of oral pathogens from the oral cavity. 


\section{Acknowledgements}

This work was partly supported by the "Promotion for Young Research Talent and Network (H20 Tal-Med-41)” fund from the Northern Advancement Center for Science \& Technology (NOASTEC) and Health and Labour Science Research grants awarded in 2006 (H18-kagaku-006) from the Ministry of Health, Labour and Welfare of Japan.

\section{References}

[1] P.T. Sarita, R. Tuominen, Proc. Finn. Dent. Soc. 88 (1992) 139-145.

[2] A.K. Camper, M.W. LeChevallier, S.C. Broadaway, A. McFeters, Appl. Environ. Microbiol. 52 (1986) 434-438.

[3] A.D. Khosravi, J. Rostamian, P. Moradinegad, J. Med. Sci. 8 (2008) 579-582.

[4] Y. Chan, C.H. Lai, Lasers Med. Sci. 18 (2003) 51-55.

[5] B.S. Lee, Y.W. Lin, J.S. Chia, T.T. Hsieh, M.H. Chen, C.P. Lin, W.H. Lan, Lasers Surg. Med. 38 (2006) 62-69.

[6] T. Akasaka, F. Watari, Y. Sato, K. Tohji, Mater. Sci. Eng. C 26 (2006) 675-678.

[7] T. Akasaka, F. Watari, Fullerenes, Nanotubes, and Carbon Nanostructure 16 (2008) $114-125$.

[8] A. Srivastava, O.N. Srivastava, S. Talapatra, R. Vajtai, P.M. Ajayan, Nat. Mater. 3 
(2004) 610-614.

[9] J.W. Kim, E.V. Shashkov, E.I. Galanzha, N. Kotagiri, V.P. Zharov, Lasers Surg. Med. 39 (2007) 622-634.

[10] V.P. Zharov, E.I. Galanzha, E.V. Shashkov, J.-W. Kim, N.G. Khlebtsov, V.V. Tuchin, J. Biomed. Opt. 12 (2007) 051503.

[11] T. Akasaka, F. Watari, Acta Biomater. 5 (2009) 607-612.

[12] S. Kang, M. Pinault, L.D. Pfefferle, M. Elimelech, Langmuir 23 (2007) 8670-8673.

[13] S. Kang, M. Herzberg, D.F. Rodrigues, M. Elimelech, Langmuir 24 (2008) 6409-6413.

[14] J.-W. Kim, E.V. Shashkov, E.I. Galanzha, N. Kotagiri, V.P. Zharov, Lasers Surg. Med. 39 (2007) 622-634.

[15] X. Zhao, S. Inoue, M. Jinno, T. Suzuki, Y. Ando, Chem. Phys. Lett. 373 (2003) 266-271.

[16] Y. Sato, A. Yokoyama, K. Shibata, Y. Akimoto, S. Ogino, Y. Nodasaka, T. Kohgo, K. Tamura, T. Akasaka, M. Uo, K. Motomiya, B. Jeyadevan, M. Ishiguro, R. Hatakeyama, F. Watari, K. Tohji, Mol. Biosyst. 1 (2005) 176-182. 


\section{Figure Captions}

(a) Macroscopic view of the CNT/agar, GP/agar, and AC/agar composites

$0.1 \mathrm{mg} / \mathrm{ml}$

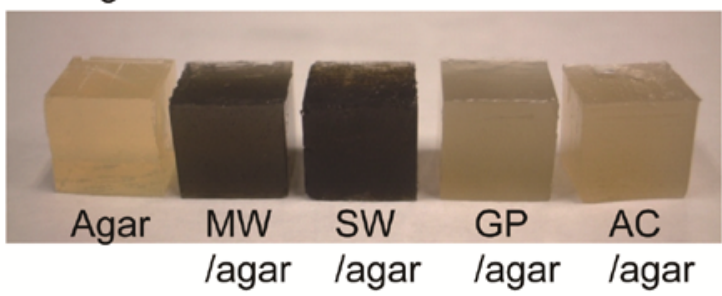

$1.0 \mathrm{mg} / \mathrm{ml}$

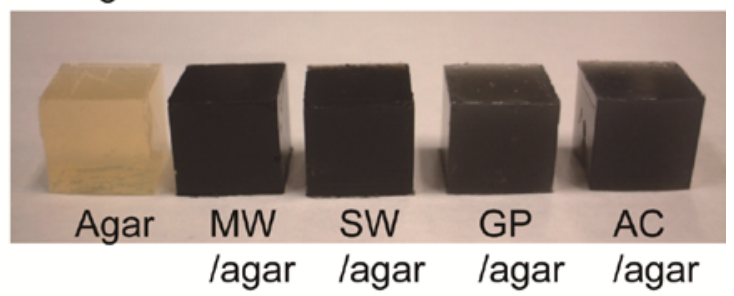

(b) Luminous transmittance of the CNT/agar, GP/agar, and AC/agar composites

\begin{tabular}{|c|c|c|c|c|c|c|c|c|c|}
\hline \multirow[b]{2}{*}{$\begin{array}{l}\text { Carbon material } \\
\text { concentration }(\mathrm{mg} / \mathrm{ml})\end{array}$} & \multirow{2}{*}{$\frac{\text { Agar }}{0}$} & \multicolumn{2}{|c|}{ MWNT/agar } & \multicolumn{2}{|c|}{ SWNT/agar } & \multicolumn{2}{|c|}{ GP/agar } & \multicolumn{2}{|c|}{ AC/agar } \\
\hline & & 0.1 & 1.0 & 0.1 & 1.0 & 0.1 & 1.0 & 0.1 & 1.0 \\
\hline Transmittance (\%) & 86.7 & 79.2 & 30.7 & 80.7 & 42.2 & 83.0 & 59.7 & 84.1 & 71.6 \\
\hline
\end{tabular}

Figure 1. Photographs of the CNT/agar, GP/agar, and AC/agar composites at concentrations of $0,0.1$, and $1.0 \mathrm{mg} / \mathrm{ml}$ (a) and their luminous transmittance (b). 


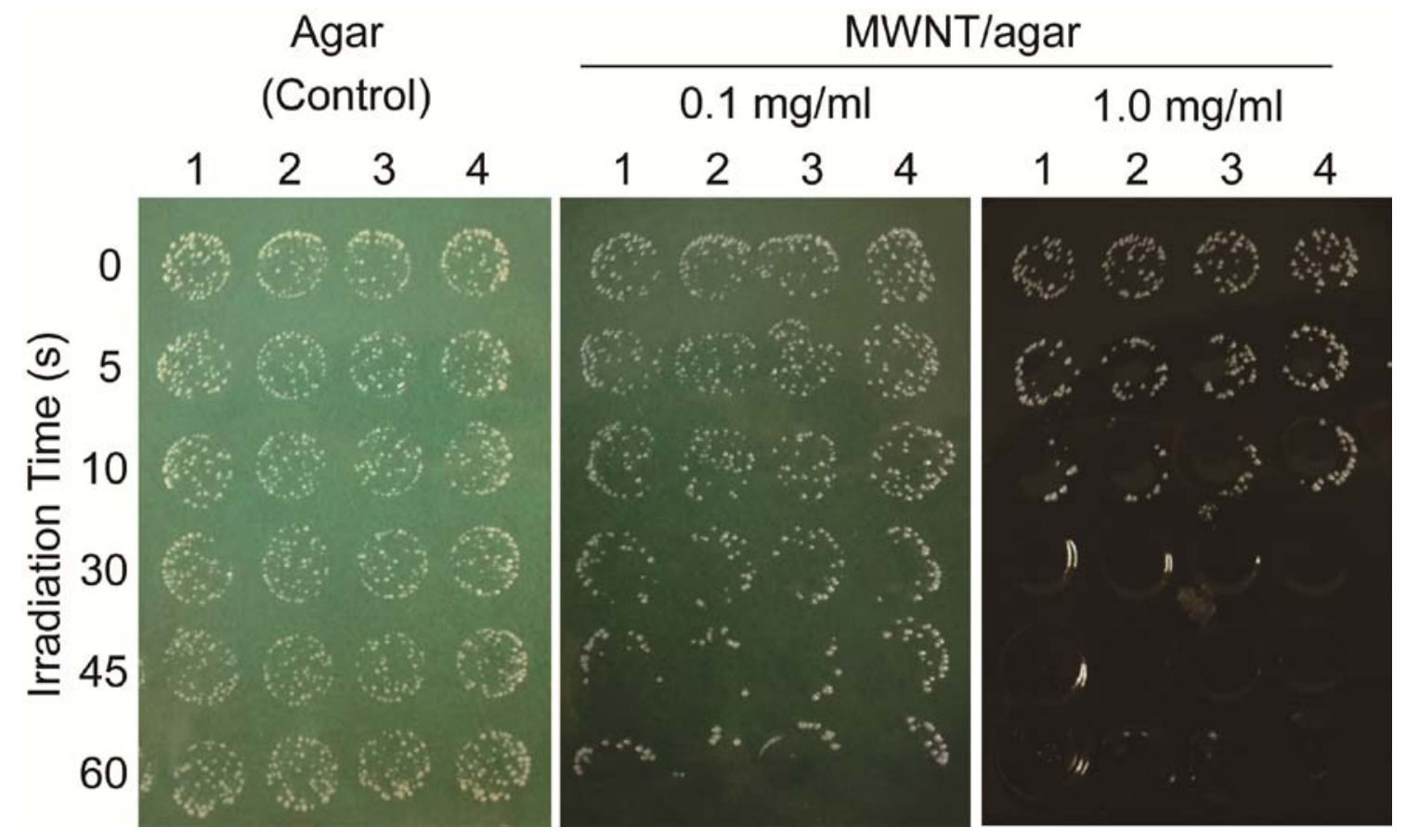

Figure 2. Results of CFU assay. Photographs of colonies on agar (control) and the MWNT/agar composites irradiated with NIR light. A 5- $\mu$ l aliquot of $6.8 \times 10^{3} \mathrm{CFU} / \mathrm{ml}$ bacterial suspension was inoculated at each spot. The NIR light power was $1.3 \times 10^{4}$ $\mathrm{W} / \mathrm{m}^{2}$. 
(a)

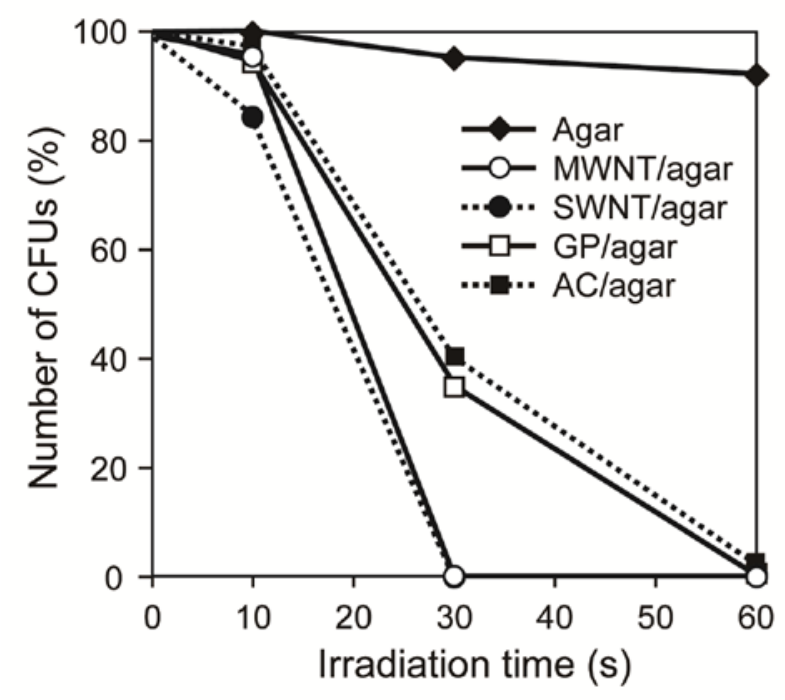

(b)

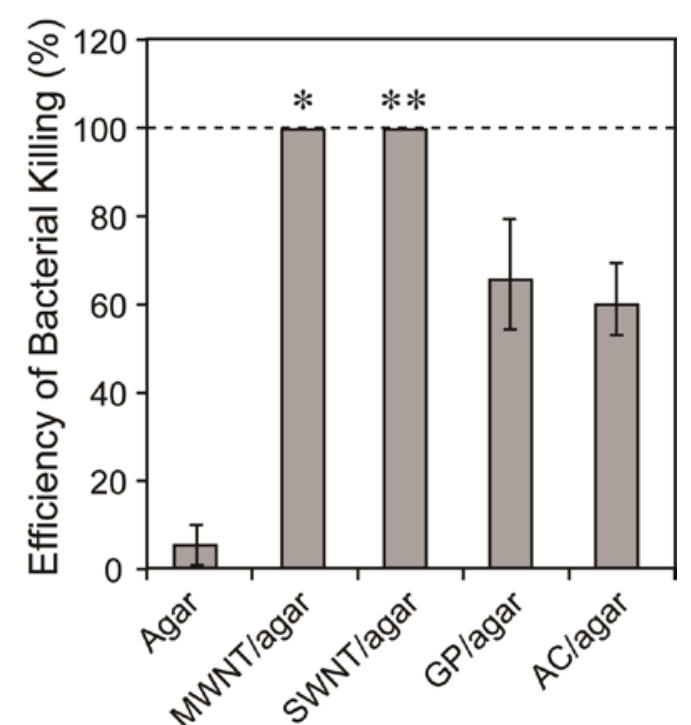

Figure 3. The bactericidal effect of various carbon/agar composites $(1.0 \mathrm{mg} / \mathrm{ml})$ irradiated with NIR light. The CFU count (a) and the bactericidal activity (\%) after 30-s irradiation (b). The NIR light power was $5.6 \times 10^{3} \mathrm{~W} / \mathrm{m}^{2}$. The percentage of CFUs was referenced against the CFUs on control agar. Results are presented as the mean \pm (standard error of the mean) (SE) of 3 experiments. ${ }^{*} p<0.05$ (compared to other agar composites except the SWNT/agar composite). ${ }^{* *} p<0.05$ (compared to other agar composites except the MWNT/agar composite). 
(A) MWNT/agar $(1.0 \mathrm{mg} / \mathrm{ml})$
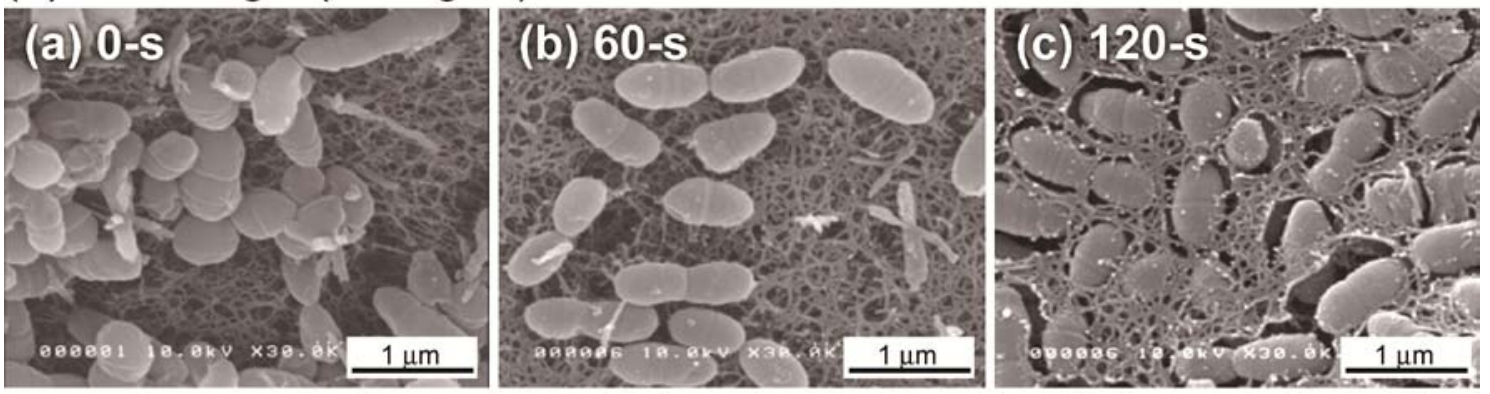

(B) Agar
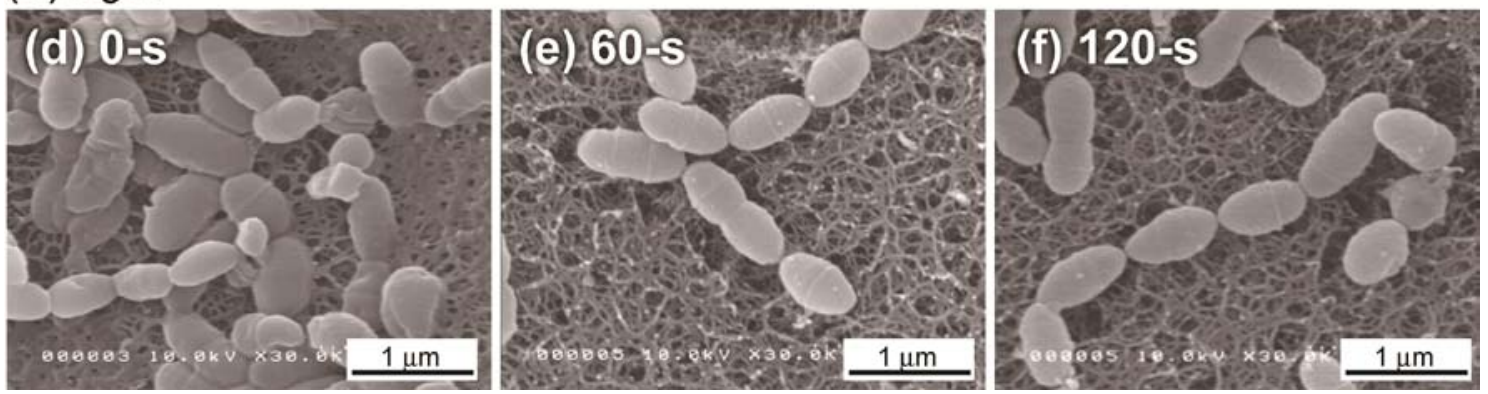

Figure 4. Typical SEM images of S.mutans on the MWNT/agar composites $(1.0 \mathrm{mg} / \mathrm{ml})$

(A) and on agar (B) irradiated with NIR light. The NIR light power was $5.6 \times 10^{3} \mathrm{~W} / \mathrm{m}^{2}$. 